\title{
Leprosy and the child in Sri Lanka
}

\section{N Atukorala}

Sri Lanka Journal of Child Health, 2003; 32: 89-91

(Key words: leprosy, children, Sri Lanka)

Sri Lanka has made much progress in the elimination of leprosy. The Multi Drug Therapy (MDT) recommended by the World Health Organisation $(\text { WHO })^{1}$ was introduced and expanded from 1982. A social marketing campaign, an awareness campaign to educate the general public about the early signs of leprosy and dispel misconceptions about the disease, was launched in $1990^{2,3,4}$. These have resulted in achieving the elimination target set by the WHO, of one case per 10,000 population for the whole country, in 1995, five years ahead of the targeted year of 2000. Since 2001, the leprosy services are being completely integrated with the general health services $^{5,6}$. The purpose of this was to enable the diagnosis and treatment of the remaining small number of cases by empowering medical officers to diagnose, treat and notify all remaining patients with leprosy. This would hopefully result in the detection and treatment of leprosy in the remaining pockets in the country, especially in the Southern, Western, Northwestern Northern and Eastern areas of the country. Thus, leprosy could be eliminated at a subnational level and it would be possible to sustain the achievements gained.

The success of the integration process will depend greatly on the ability and desire of all doctors to diagnose and treat patients with leprosy. This would need a sustained education and motivation programme. The distribution of the necessary drugs and materials for notification and follow up and the coordination of the efforts at sub national level via the regional epidemiologists, with the aid of the local dermatologists and provincial directors, will be vital.

The new case detection rate per 10,000 population, which had dropped to 7 in 1998, showed an increase to 12 in 2001 with the introduction of integration programme. This number is slowly decreasing in $2003^{7}$. Thus, new cases are being detected with the new programme. The percentage of children among all new cases was around 10\% in 1998 and has fallen to $8 \%$ (162 cases) in 2003 . In a recent study many of

\footnotetext{
${ }^{1}$ Consultant Dermatologist
}

these children were found to be contacts of diagnosed adult cases ${ }^{7}$. It is vital to diagnose correctly all new child cases of leprosy, as it indicates recent transmission and since a gross increase would indicate that the elimination strategy is failing.

Leprosy is a disease of the skin and nerves caused by the Mycobacterium leprae. This is a very slow growing bacterium and hence the disease, which spreads by droplet inhalation, progresses very slowly and has a long incubation period. Leprosy can now be cured. MDT kills the bacteria and stops spread of the disease. Leprosy patients, once treatment is started, can lead completely normal lives. They can live at home, go to school, play, and participate in social events. If detected early and treated, leprosy will not lead to any disabilities or deformities. The best way to prevent the spread of leprosy is to diagnose and treat all patients. There is no vaccine for leprosy.

Leprosy can be diagnosed by clinical signs alone. The presence of hypopigmented, well demarcated or slightly reddish patch or patches with a definite or significant loss of sensation and /or signs of nerve damage, in the form of a definite loss of sensation in the hands and feet or a weakness of muscles of the hands, feet or face would indicate a diagnosis of leprosy. Thickening of nerves in appropriate locations, presence of erythematous or skin coloured papules, plaques and nodules, especially on the face, are further signs.

Care must be taken to ensure that all cases diagnosed do fit these signs. Hypopigmented patches, such as pityriasis alba, and those of post inflammatory pigmentation, are especially common in the skin of a child. It is essential to realize that skin patches need to have a significant loss of sensation to be diagnosed as leprosy. Patches present from around the time of birth are achromic naevi. Itchy patches, completely depigmented patches, scaly patches and those that appear suddenly and spread fast are not those of leprosy. 
Leprosy has been traditionally classified into tuberculoid, borderline and lepromatous. It is in fact a spectrum of disease depending on many factors, the chief of which is host cell mediated resistance to leprosy. TH1 responses in pauci-bacillary (PB), and TH2 responses in multi-bacillary (MB), found by cytokine studies may be further factors ${ }^{8}$. For treatment purposes, the WHO classification into $\mathrm{PB}$ (patients with 1-5 patches), and MB (those with more than 5 patches), is adequate ${ }^{9}$. This classification also avoids the need for split skin smears except in special circumstances. Most childhood cases of leprosy will be of the PB type (22\%).

MDT therapy in PB leprosy consists of rifampicin $600 \mathrm{mg}$ once per month and dapsone $100 \mathrm{mg}$ daily for 6 months. The therapy for MB leprosy consists of rifampicin $600 \mathrm{mg}$ once per month, clofazimine 300 $\mathrm{mg}$ once per month and $50 \mathrm{mg}$ daily and dapsone 100 $\mathrm{mg}$ daily for a period of 12 months. Various views have been expressed particularly on the duration of the regimes. However many studies have shown the validity of these regimes ${ }^{10,11,12}$.

The urine becomes red on the day of ingestion of rifampicin. The skin gets dark on treatment with clofazimine. These are normal and the latter will revert to normal a few months after the completion of therapy. Side effects to therapy are present but fortunately rare. Hepatitis, haemolysis leading to renal failure, and cutaneous hypersensitivity reactions are the main serious side effects. A degree of haemolysis may be present with dapsone but this usually recovers.

Lepra reactions are inflammatory episodes in leprosy, before, during or after treatment. They are not side effects of the drugs. In Type 1 reactions, a cell mediated hyper-reactivity, the skin patches of leprosy become swollen and the associated nerves thick and tender, leading to loss of sensory and motor function of the localized area. Type 2, or erythema nodosum leprosum reactions are immune complex mediated reactions, commoner at the lepromatous end of the disease spectrum, and are characterized by numerous new red tender patches and nodules, thickened and tender nerves and loss of function of the nerves. In a retrospective study in Sri Lanka, 6.6\% of all treated patients had lepra reactions - 80\% were type $1,15 \%$ were type 2 and $5 \%$ were mixed type 1 and 2 reactions ${ }^{13}$. The therapy for reactions is the use of high dosage prednisolone, with the continuation of MDT therapy. The recommended WHO dosage is a starter dose of $40 \mathrm{mg}$ per day to be reduced gradually and omitted in around 3 months. The addition of analgesics and increase in the dose of clofazimine are additional measures in therapy.

Deformities are still stigmata of leprosy. The trophic ulcers due to sensory loss, the motor deformities of a claw hand, foot drop or a facial paralysis and the eye lesions of leprosy all need early recognition and care.

In any chronic infectious disorder there must be adequate new drugs, in case resistance develops to the standard drugs or for more effective therapy. Dapsone resistance, both partial and total, is well recognized, though the dose of dapsone used in the standard WHO regime is adequate to overcome any partial resistance. Rifampicin resistance is still very rare $^{15}$, and no clofazimine resistance is yet reported. New drugs are now available. Oflxacin, minocycline and clarythromycin have all been found effective though all less bactericidal than rifampicin. Moxifloxin is as effective as rifampicin and rifapentine is the most bactericidal drug against $M$. Leprae. Trials are under way with combinations of these drugs ${ }^{16}$.

In areas of high HIV prevalence the prevalence of leprosy has shown no increase and the standard treatment regimes have proved successful. However a highly significant association between HIV positivity and the development of neuritis and reactions have been shown.

PCR has still not been successful as a diagnostic tool, though resistance to drugs have been demonstrated using PCR probes. Vaccines have still not proved of use.

The eradication of leprosy will also require an alleviation of the living standards of the poor of this country ${ }^{14}$. It should be remembered that leprosy was eradicated from the developing west even before the advent of dapsone. However, our contribution can only be the correct diagnosis and management of all patients with leprosy in the country.

\section{References}

1. WHO study group. Chemotherapy of leprosy for control programmes. 1982. WHO Tech.Rep.Ser.no 675 World Health Organisation. Geneva.

2. Devapura D. Anti Leprosy Campaign. Annual Administration Report 1988.

3. Devapura D R. The current state of leprosy control activities in Sri Lanka. Leprosy Review 1989; 60: 39-44. 
4. Salgado S. Eliminating leprosy from Sri Lanka - the launch of a social marketing campaign. Ceylon Medical Journal 1993; 38:95-7.

5. Settinayake S. 2001. Country report 20002001. Inter-country meeting of National programme managers for elimination of leprosy. 2001.

6. Settinayake S. Integrating leprosy services into the general health care system in Sri Lanka. Sri Lanka Journal of Dermatology 2001; 5: 2-4.

7. Settinayake S. Personal communication

8. Sasaki S, Takeshita F, Okuda K, Ishii N. Microbiol Immunol 2001:45: 729-38.

9. Action Programme for the Eliminatipn of Leprosy. A guide to eliminating leprosy as a public heath problem. 1995; World Health Organization; WHO/LEP/95.1.

10. WHO. A guide to eliminate leprosy as a public health problem 2000; World Health Organisation, 2000.
11. AI-Qubati Y. Multidrug therapy: the pathway for global elimination. Indian Journal of Leprosy 2000; 72: 477-90.

12. Danmerie D. Current World Health Organisation sponsored studies in the chemotherapy of leprosy. Lepr Rev. 2000: 71 88-90.

13. Ragunathan $\mathrm{R}$ W, Atukorala $\mathrm{D} N$, Gunawardena P. Leprosy - A study of reactions in Sri Lanka. Abstracts; South Asian Regional Conference of Dermatology, Venereology. and Leprology, 2003: 52.

14. Kumarasinghe S P W. Leprosy in Sri Lanka. Sri Lanka Journal of Dermatology 2001; 5:1-2.

15. Ji.B. Rifampicin resistant leprosy: A review and a research proposal of a pilot study. Leprosy Review 2002; 73: 2-8.

16. TDR technical committee. New and improved methods for treatment. TDR progress 1999-2000; 1 -2 